\title{
A graphical method to aid the sequential analysis of observational data
}

\author{
SCOTT HALL and CHRIS OLIVER \\ University of Birmingham, Birmingham, England
}

\begin{abstract}
In this paper, two methods of sequential analysis are applied to hypothetical observational data. The first method employs the conventional "conditional probability" approach, illustrated using the GSEQ program (Bakeman \& Quera, 1995). In order to overcome some of the difficulties associated with the conditional probability approach, the second method employs a new "normalized and pooled" approach. Essentially, by normalizing periods of time preceding, during, and following each occurrence of a nominated "given" behavior, the proportion of time units devoted to a "target" behavior can be estimated and then pooled across all occurrences of the given behavior. A summary diagram representing the likelihood that the target behavior precedes, occurs concurrently with, and follows the given behavior can then be constructed. Elements of this summary diagram can also be quantified. Given the graphical nature of the output, and its ease of use, the normalized and pooled approach may help to promote the use of sequential analysis in applied settings.
\end{abstract}

The analysis of complex social interactions has attracted increasing interest in the last 15 years. Prominent targets for study have included the mother-child relationship (Adamson \& Bakeman, 1985; Bakeman \& Brown, 1977; Dowdney, Mrazek, Quinton, \& Rutter, 1984; Gottman \& Ringland, 1981), husband-wife interactions (Gottman, 1979; Gottman, Markman, \& Notarius, 1977), the client-counselor process (Hill, Carter, \& O'Farrell, 1983; Lichtenberg \& Heck, 1986; Patterson \& Forgatch, 1985), and, more recently, the relationship between problem behaviors and environmental events (Lerman \& Iwata, 1993; Mace \& Lalli, 1991). In an attempt to quantify various aspects of interactions, many studies have employed observational methodology. This involves systematically observing and recording the behaviors of two or more individuals, usually in naturalistic settings. Given computerized methods of data capture, this has now become a less onerous task than it once was (see Noldus, 1991; Repp \& Felce, 1990; Repp, Harman, Felce, Van Acker, \& Karsh, 1989; Tapp, Wehby, \& Ellis, 1995; Unwin \& Martin, 1987). For instance, behaviors can now be recorded in either "event" or "interval" units, be defined as "momentary" and/or "duration" (see Sackett, 1979), and saved in either a "continuous" or an "intermittent" format. The data can then be subjected to analysis.

Although each recorded behavior can be expressed as a rate or percentage of the total observation period, these

This research was supported by a grant from the Mental Health Foundation, U.K. The authors thank Gian Jones for his help with the "normalized and pooled" software program and thank Glyn Murphy for her comments on an earlier draft of this manuscript. The software was developed using computer equipment generously donated by Viglen Limited. Correspondence should be addressed to C. Oliver, School of Psychology, University of Birmingham, Edgbaston, Birmingham B15 2TT, England (e-mail: oliverc@psychol.bham.ac.uk). "time-budget" measures do not provide information concerning the process of the interaction (Bakeman \& Gottman, 1986) - that is, the relationship between behaviors. Many studies have therefore attempted to detect naturally occurring chains of behaviors in the observational data (see Patterson, 1974). However, a simple, yet effective and useful methodology for analyzing sequential data has proved elusive (Gottmian \& Roy, 1990). Previous descriptions of methods of sequential analysis (e.g., Budescu, 1984; Gardner \& Griffin, 1989; Gottman, 1981; Iacobucci \& Wasserman, 1988; Moran, Dumas, \& Symons, 1992) and software for conducting sequential analysis (e.g., Noldus, 1991; Repp \& Felce, 1990; Sackett, Holm, Crowley, \& Henkins, 1979) require considerable time and statistical expertise in terms of conducting the analysis itself and interpreting the subsequent output (Bakeman \& Casey, 1995; Hall, 1995; Highlen, 1986; Lichtenberg \& Heck, 1986; Sackett, 1987). Sequential analysis has therefore not been utilized by practicing behavior analysts in clinical settings.

Some of the difficulties with sequential analysis have now been resolved. For instance, Bakeman and Quera (1992) have developed a standard format for representing observational data collected using different methods (i.e., the sequential data interchange standard, or SDIS). A software package for data analysis has also recently appeared (i.e., the Generalized Sequential Querier, or $G S E Q$, Bakeman \& Quera, 1995). However, some difficulties with the application and interpretation of sequential analysis methods remain. In order to illustrate these difficulties, a hypothetical example will be considered, and a new graphical method of sequential analysis will then be described. The new method is designed primarily to help promote the use of sequential analysis by practicing behavior analysts in clinical settings and not as a replacement for existing methods of sequential analysis. 


\section{Example Data}

Suppose two behaviors, $\mathrm{X}$ and $\mathrm{Y}$, were observed in a naturalistic setting. Behavior $X$, for instance, could represent the "gaze" of a young child, whereas behavior Y could represent the "vocalization" of his/her mother. Hypothetical data for the dyad are displayed at the bottom of this page. ${ }^{1}$ These data follow SDIS conventions (Bakeman \& Quera, 1995). The first line in the data (i.e., Timed $X \mathrm{Y}$;) indicates that two behavior codes were recorded in continuous mode. The second line (i.e., , l) indicates that the observation session started at the first time unit. The following lines contain the main observational data. Each recorded behavior occurrence has three components: a letter indicating which behavior was emitted, its onset time, and its subsequent offset time. The right parenthesis following each offset time unit indicates an inclusive offset time unit (see Bakeman \& Quera, 1995). Inspection of the data above shows that behaviors $\mathrm{X}$ and $Y$ can co-occur (e.g., at time units 100 and 101). The last line in the data (i.e., ,547)/) indicates that the observation session finished at time unit 547. This particular data format has been called timed sequential data (TSD) and is considered to capture "real-life complexity" (Bakeman \& Quera, 1995, p. 14).

\section{The "Conditional Probability" Approach}

Conventional methods of sequential analysis adopt the "transitional" or "conditional probability" approach (Bakeman, 1978). The conditional probability of a "target" behavior reflects the likelihood of its occurrence given that another "given" behavior has occurred. Thus, in the example data shown above, the probability of $Y$ given $\mathrm{X}$, or $p(\mathrm{Y} / \mathrm{X})$, and the probability of $\mathrm{X}$ given $\mathrm{Y}$, or $p(\mathrm{X} / \mathrm{Y})$, are statistical descriptions of the association between X and Y. Quantification of the association can most easily be achieved by comparing each conditional probability with its respective unconditional probability (i.e., the proportion of time allocated to the behavior). Thus, a higher (or lower) value obtained for the probability of $Y$ given $X$, when compared with the unconditional probability of $Y$, indicates a likely association between $X$ and Y. Similarly, a higher (or lower) value obtained for the probability of X given $Y$, when compared with the unconditional probability of $\mathrm{X}$, also indicates a likely association between $\mathrm{X}$ and $\mathrm{Y}$. The magnitude of the effect can be gauged using an appropriate statistic-for example, Yule's $Q$ or the log-odds ratio (Wickens, 1993).

The temporal association between $\mathrm{X}$ and $\mathrm{Y}$ (i.e., whether the target behavior precedes, occurs during, or follows the given behavior) can be indicated by adding a subscript to the notation given above. Thus, to evaluate the temporal association between $\mathrm{X}$ and $\mathrm{Y}$, the probability that $Y$ precedes $X$ could be represented by the notation $p\left(\mathrm{Y}_{-1} / \mathrm{X}\right)$, the probability that $\mathrm{Y}$ occurs during $\mathrm{X}$ could be represented by the notation $p\left(\mathrm{Y}_{+0} / \mathrm{X}\right)$, and the probability that $Y$ follows $X$ could be represented by the notation $p\left(\mathrm{Y}_{+1} / \mathrm{X}\right)$. Similarly, the probability that $\mathrm{X}$ precedes $\mathrm{Y}$ could be represented by the notation $p\left(\mathrm{X}_{-1} / \mathrm{Y}\right)$, the probability that $\mathrm{X}$ occurs during $\mathrm{Y}$ could be represented by the notation $p\left(\mathrm{X}_{+0} / \mathrm{Y}\right)$, and the probability that $\mathrm{X}$ follows $\mathrm{Y}$ could be represented by the notation $p\left(\mathrm{X}_{+1} / \mathrm{Y}\right)$. Thus, six conditional probabilities can be evaluated to reflect the association between $\mathrm{X}$ and $\mathrm{Y}$.

To illustrate the conventional conditional probability approach to sequential analysis, an analysis will be conducted on the hypothetical data given above using the GSEQ program. GSEQ is a command-based program that requires the user to create an ASCII input file of commands. For instance, in order for the program to calculate the conditional probability of $\mathrm{Y}$ occurring during $\mathrm{X}$ that is, $p\left(\mathrm{Y}_{+0} / \mathrm{X}\right)$ the following commands would be required in the GSEQ input file:

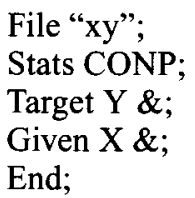

The first line (i.e., File "xy";) indicates that the data file is called xy.sds. The second line (i.e., Stats CONP;) requests conditional probability values. The third and fourth lines (i.e., Target Y \&; and Given X \&;) request conditional probability values for the target behaviors, $Y$ and not-Y (the latter represented by $\&$ ), given the given behaviors $X$ and not-X. The last line (i.e., End;) finishes the program run. The conditional probability values actually calculated by GSEQ are simply the number of time units during which the target and given behaviors co-occur divided by the number of time units during which the respective given behaviors occur (cf. "nested" analysis; Noldus, 1991; Repp \& Felce, 1990). For instance, if X occurs for 120 time units and $X$ and $Y$ co-occur for 35 time units, then the conditional probability of $Y$ occurring during $X$ is .29 (i.e., 35 divided by 120 ). However, the calculation of conditional probabilities for $\mathrm{Y}$ preceding $\mathrm{X}$, or for $\mathrm{Y}$ following $X$, is more difficult. Here, GSEQ allows the user to specify time "windows" (i.e., periods of time keyed to the onset or offset of behaviors). Time windows are essen-

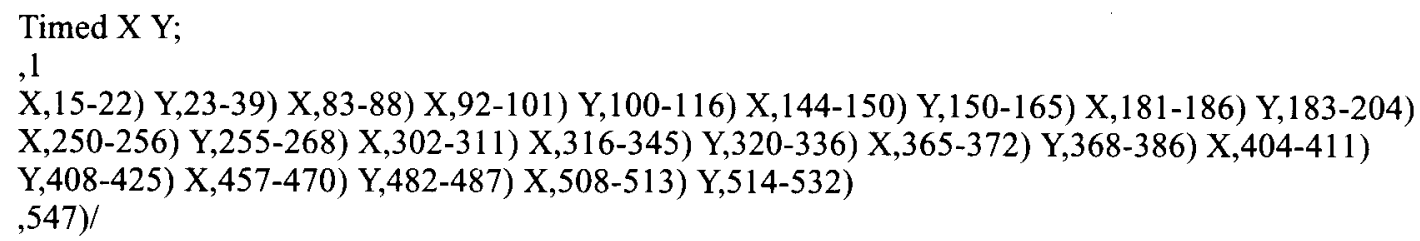


tially additional variables that are created at run time and can therefore be used in the subsequent analysis. For example, issuing the commands:

Window PREX $=(X-10,(X-1$;

Window FOLX $=X)+1, X)+10$;

after the first line in the GSEQ input file given above generates two new variables, PREX and FOLX. The first variable, PREX, would occur 10 time units before the onset of each occurrence of $X$ to 1 time unit before the onset of each occurrence of X. The second variable, FOLX, would occur 1 time unit after the offset of each occurrence of $X$ to 10 time units after the offset of each occurrence of $X$. Exchanging $X$ in the GSEQ input file given above for PREX or FOLX would then allow the program to calculate the conditional probability of $Y$ preceding $X$ or the conditional probability of $Y$ following $X$. One disadvantage of the window command, however, concerns the problem of "overlapping" time windows. For instance, by specifying a time window of $10 \mathrm{sec}$, a period of $10 \mathrm{sec}$ after the offset of a given behavior may overlap with a period $10 \mathrm{sec}$ before the onset of the next given behavior, or even with the next occurrence of the given behavior. As Bakeman and Quera (1995) explain:

The windows ... are affected by the timing of the codes already in the file, and occasionally this very flexibility can result in anomalies. Some windows may extend before the beginning or after the end of the session, in which case they are truncated. Others may overlap, in which case the later windows are truncated. Still others may be null (i.e., have no duration). (p. 76)

Employing a 10-sec time window on the example data given above would produce an overlapped time window (i.e., the window following " $X, 83-88$ )" would overlap with the window preceding "X,92-101)"). Although this would have a minimal effect on the interpretation of the results in this example, anomalies may become substantial on larger data sets, particularly when behaviors cluster together (i.e., they have short bout and interbout durations). Thus, it appears that an analysis employing a time window would be entirely satisfactory only if the periods of time between occurrences of a behavior were always more than twice the desired time window. Since this is unlikely (unless the time window specified is extremely short), the analysis process requires the investigator to make a "decision" concerning the length of the time window in order to minimize the anomalies. Clearly, different specifications will produce quite different results.

Still, there is a solution to the overlap problem within this approach. For example, when the time between the offset of a given behavior and the onset of its next occurrence is less than twice the desired window (e.g., $10 \mathrm{sec}$ ), then whatever time is available can be split - the first half assigned to time after the first behavior, and the sec- ond half assigned to time before the next occurrence. ${ }^{2}$ Therefore, once GSEQ has issued a warning that a window variable has created overlaps, the window variable could be coded manually so that the time between any overlaps is split. For instance, in the example data, given that GSEQ flagged an overlap for the window variable PREX, the variables PREX and FOLX were coded manually (using the splitting procedure for the one overlap) and placed in the data file before running GSEQ. Due to the work involved, however, this procedure would seldom be done without the aid of a computer program. Still, the window command in GSEQ can be used to define the variables PREY and FOLY (i.e., 10-sec time periods preceding and following $Y$ ) since these variables do not overlap (at least with the $10-\mathrm{sec}$ window used here). The input GSEQ command file and corresponding data file necessary to conduct this "modified" analysis are shown in the Appendix. Figure 1 shows selected parts of output given by GSEQ.

Each $2 \times 2$ table in Figure 1 contains conditional probability data for a target behavior given a given behavior or created window variable. For instance, the table at the top left of Figure 1 shows that the conditional probability of $\mathrm{Y}$ preceding $\mathrm{X}$, or $p\left(\mathrm{Y}_{-1} / \mathrm{X}\right)$, is .00 (i.e., the value in the top left cell of the table). Yule's $Q$ for the table is -1.00 . It can therefore be stated that $Y$ is less likely to occur preceding $X$. Looking at the middle left table in Figure 1, we can see that the conditional probability of $Y$ during $\mathrm{X}$, or $p\left(\mathrm{Y}_{+0} / \mathrm{X}\right)$, is .29 (Yule's $Q=-.03$ ). Thus, $\mathrm{Y}$ is neither less likely nor more likely to occur during $\mathrm{X}$. The conditional probability of $\mathrm{Y}$ following $\mathrm{X}$, or $p\left(\mathrm{Y}_{+1} / \mathrm{X}\right)$, can be found in the bottom left table in Figure 1. Here, the conditional probability is .77 (Yule's $Q=.87$ ). Thus, $\mathrm{Y}$ is more likely to occur following $\mathrm{X}$. Interpreting these results in terms of the example behavior codes (i.e., $X$ representing child gaze and $Y$ representing mother vocalize), it appears that mother vocalize occurs at a lower level prior to child gaze and at a higher level following child gaze.

The top right table in Figure 1 shows that the conditional probability of $\mathrm{X}$ preceding $\mathrm{Y}$, or $p\left(\mathrm{X}_{-1} / \mathrm{Y}\right)$, is .48 (Yule's $Q=.66$ ). Thus, $\mathrm{X}$ is more likely to occur preceding $Y$. The middle right table in Figure 1 shows that the conditional probability of $\mathrm{X}$ during $\mathrm{Y}$, or $p\left(\mathrm{X}_{+0} / \mathrm{Y}\right)$, is .21 (Yule's $Q=-.03$ ). Thus, $\mathrm{X}$ is neither less likely nor more likely to occur during $Y$. The bottom right table in Figure 1 shows that the conditional probability of $\mathrm{X}$ following $\mathrm{Y}$, or $p\left(\mathrm{X}_{+1} / \mathrm{Y}\right)$, is .09 (Yule's $Q=-.54$ ). Thus, $\mathrm{X}$ is less likely to occur following $Y$. Again, interpreting these results in terms of the example behavior codes, it appears that child gaze occurs at a higher level prior to mother vocalize and at a lower level following mother vocalize. In summary then, the six tables presented in Figure 1 tell the same story (i.e., they are six views of the same data) - specifically, that mother vocalize often followed child gaze. 


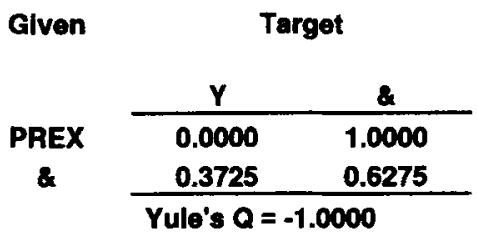

Given

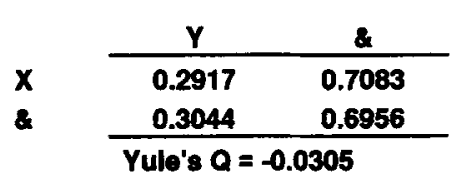

Given

\begin{tabular}{ccc} 
& $Y$ & \multicolumn{1}{c}{$\&$} \\
\cline { 2 - 3 } FOLX & 0.7692 & 0.2308 \\
$\&$ & 0.1919 & 0.8081 \\
\cline { 2 - 3 } & Yule's $Q=0.8670$
\end{tabular}

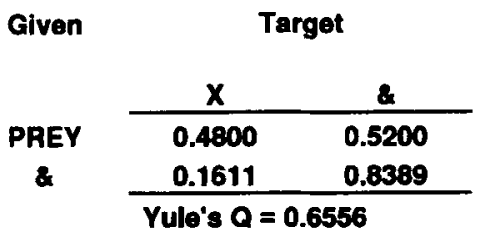

Given

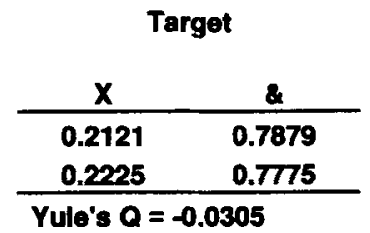

Given

Target

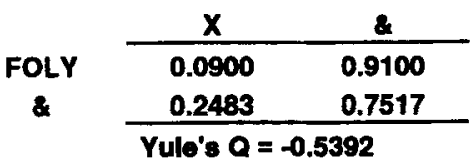

Figure 1. Selected output obtained from the GSEQ program following issue of the commands and modified example data listed in the Appendix. Cells in the tables contain conditional probabilities. The Yule's $Q$ statistic is shown below each table.

\section{The "Normalized and Pooled" Approach}

In order to overcome some of the difficulties encountered when conducting the conditional probability approach and to provide a useful graphical aid to sequential analysis, a "normalized and pooled" approach is proposed. ${ }^{3}$ The method proceeds as follows. Each $i$ th occurrence of a given behavior (e.g., X) is examined in turn, beginning at the 2nd occurrence of the given behavior and ending at its penultimate occurrence ${ }^{4}$ Three "periods," consisting of time units, are then imposed on the data: ${ }^{5}$ (1) preceding (from the midpoint between the time unit following the offset of the $i$ th -1 occurrence and the time unit before the onset of the $i$ th occurrence, denoted $a_{1}$, to the time unit before the onset of the $i$ th occurrence, denoted $b_{1} ;(2)$ during (from the onset of the $i$ th occurrence, denoted $a_{2}$, to its offset inclusive, denoted $b_{2} ;$ and (3) following (from the time unit following the offset of the $i$ th occurrence, denoted $a_{3}$ to the midpoint between $a_{3}$ and the time unit before the onset of the $i$ th +1 occurrence, denoted $b_{3}$ ). The third column of Figure 2 shows the time units for the three periods: preceding, during, and following each $i$ th occurrence of $\mathrm{X}$ in the example data.

Next, for each $i$ th occurrence of the given behavior, the time units at which a target behavior (e.g., Y) co-occurs in each period is noted. If a target behavior co-occurs in a preceding period, its onset time unit is denoted $c_{1}$ and its offset time unit is denoted $d_{1}$. If a target behavior cooccurs in a during period, its onset time unit is denoted $c_{2}$ and its offset time unit is denoted $d_{2}$. Finally, if a tar- get behavior co-occurs in a following period, its onset time unit is denoted $c_{3}$ and its offset time unit is denoted $d_{3}$. The fourth column of Figure 2 shows the time units at which the target behavior Y co-occurs in each of the three periods for each $i$ th occurrence of the given behavior $\mathrm{X}$ in the example data.

Because each period consists of differing time unit lengths, each period is normalized in order to produce time periods of equal length, and the proportion of time units at which the target behavior co-occurs in each of the three periods of each $i$ th occurrence of the given behavior is calculated. This is done using the following equation:

$$
\begin{aligned}
\text { percentiles }= & \frac{c_{x}-a_{x}}{\left(b_{x}-a_{x}\right)+1} \\
& \times 100 \text { to } \frac{\left(d_{x}-a_{x}\right)+1}{\left(b_{x}-a_{x}\right)+1} \times 100,
\end{aligned}
$$

where $x=1,2$, or 3 (i.e., preceding, during, or following periods, respectively). The fifth column of Figure 2 shows the resulting percentile data for each period and each occurrence of the given behavior $\mathrm{X}$ in the example data.

To illustrate, for the 3rd occurrence of $\mathrm{X}$ in the example data, the time units for the following period are 102 to 122 , (therefore, $a_{1}=102$, and $b_{1}=122$; see Figure 2, column 3 ). The time units at which $\mathrm{Y}$ co-occurs in the period are 102 to 116 , (therefore, $c_{1}=102$, and $d_{1}=116$; see Figure 2, column 4). Using the equation above, 


\begin{tabular}{|c|c|c|c|c|}
\hline $\begin{array}{l}\text { Ah } \\
\text { occurrence } \\
\text { of } X\end{array}$ & Period & $\begin{array}{l}\text { Time units for each } \\
\text { period }\end{array}$ & $\begin{array}{l}\text { Co-occurrence of } Y \\
\text { In each period }\end{array}$ & $\begin{array}{l}\text { Percentiles for each } \\
\text { perlod }\end{array}$ \\
\hline 2 & $\begin{array}{l}\text { Proceding } \\
\text { During } \\
\text { Following }\end{array}$ & $\begin{array}{l}53-82) \\
83-88) \\
89-90)\end{array}$ & & \\
\hline 3 & $\begin{array}{l}\text { Proceding } \\
\text { During } \\
\text { Following }\end{array}$ & $\begin{array}{c}90-91) \\
92-101) \\
102-122)\end{array}$ & $\begin{array}{l}100-101) \\
102-116)\end{array}$ & $\begin{array}{c}80 \text { to } 100 \\
0 \text { to } 71\end{array}$ \\
\hline 4 & $\begin{array}{l}\text { Preceding } \\
\text { During } \\
\text { Following }\end{array}$ & $\begin{array}{l}123-143 \text { ) } \\
144-150 \text { ) } \\
151-165)\end{array}$ & $\begin{array}{c}150 \\
151-165)\end{array}$ & $\begin{array}{l}86 \text { to } 100 \\
0 \text { to } 100\end{array}$ \\
\hline 5 & $\begin{array}{l}\text { Preceding } \\
\text { During } \\
\text { Following }\end{array}$ & $\begin{array}{l}166-180) \\
181-186) \\
187-218)\end{array}$ & $\begin{array}{l}183-186) \\
187-204)\end{array}$ & $\begin{array}{c}33 \text { to } 100 \\
0 \text { to } 56\end{array}$ \\
\hline 6 & $\begin{array}{l}\text { Preceding } \\
\text { During } \\
\text { Following }\end{array}$ & $\begin{array}{l}218-249) \\
250-256 \text { ) } \\
257-279)\end{array}$ & $\begin{array}{l}255-256) \\
257-268)\end{array}$ & $\begin{array}{c}71 \text { to } 100 \\
0 \text { to } 52\end{array}$ \\
\hline 7 & $\begin{array}{l}\text { Proceding } \\
\text { During } \\
\text { Following }\end{array}$ & $\begin{array}{l}\text { 279-301) } \\
302-311) \\
312-313)\end{array}$ & & \\
\hline 8 & $\begin{array}{l}\text { Proceding } \\
\text { During } \\
\text { Following }\end{array}$ & $\begin{array}{l}314-315 \text { ) } \\
316-345 \text { ) } \\
346-355)\end{array}$ & $320-336)$ & 13 to 70 \\
\hline 9 & $\begin{array}{l}\text { Preceding } \\
\text { During } \\
\text { Following }\end{array}$ & $\begin{array}{l}355-364) \\
365-372 \text { ) } \\
373-388 \text { ) }\end{array}$ & $\begin{array}{l}368-372) \\
373-386)\end{array}$ & $\begin{array}{c}38 \text { to } 100 \\
0 \text { to } 88\end{array}$ \\
\hline 10 & $\begin{array}{l}\text { Preceding } \\
\text { During } \\
\text { Following }\end{array}$ & $\begin{array}{l}388-403) \\
404-411) \\
412-434)\end{array}$ & $\begin{array}{l}408-411) \\
412-425)\end{array}$ & $\begin{array}{c}50 \text { to } 100 \\
0 \text { to } 61\end{array}$ \\
\hline 11 & $\begin{array}{l}\text { Preceding } \\
\text { During } \\
\text { Following }\end{array}$ & $\begin{array}{l}434-456) \\
457-470) \\
471-489)\end{array}$ & 482-487) & 58 to 90 \\
\hline
\end{tabular}

Figure 2. Time units for the three "periods" preceding, during, and following each $i$ th occurrence of $X$, time units for the co-occurrence of $Y$ in each period, and percentiles of time units for the co-occurrence of $Y$ in each period in the example data.

$$
\begin{aligned}
\text { percentiles } & =\frac{102-102}{(122-102)+1} \\
& \times 100 \text { to } \frac{(116-102)+1}{(122-102)+1} \times 100=0 \text { to } 71 .
\end{aligned}
$$

The percentiles at which $Y$ co-occurs in the following period are therefore 0 to 71 (i.e., $Y$ co-occurs in the first 72 percentiles of the period; see Figure 2, column 5).
In order to calculate the mean probability of a target behavior occurring at a given percentile for each period of the given behavior, the total number of occurrences of the target behavior occurring at each percentile is pooled across all occurrences of the given behavior for each period. To illustrate, in the example data, behavior $\mathrm{Y}$ cooccurs at percentile 0 in the following period of the $3 \mathrm{rd}$, 4 th, 5th, 6th, 9th, and 10th occurrences of X. The total number of pooled occurrences of $Y$ occurring at per- 
centile 0 of the following period is therefore 6 . The total number of occurrences of the target behavior occurring at each percentile is then divided by the total number of occurrences of the given behavior considered (i.e., if $\mathrm{X}$ is nominated the given behavior in the example data, the total number of occurrences of the target behavior $Y$ occurring at each percentile would be divided by 10). Considering the example again, the probability of $Y$ occurring at percentile 0 in the following period would thus be .6 .

A summary diagram of the probability of the target behavior preceding, occurring during, and following the given behavior can now be constructed. The resulting diagrams for the example data with $\mathrm{X}$ as the given behavior and $\mathrm{Y}$ as the target behavior (upper panel) and with $\mathrm{Y}$ as the given behavior and $\mathrm{X}$ as the target behavior (lower panel) are shown in Figure 3.
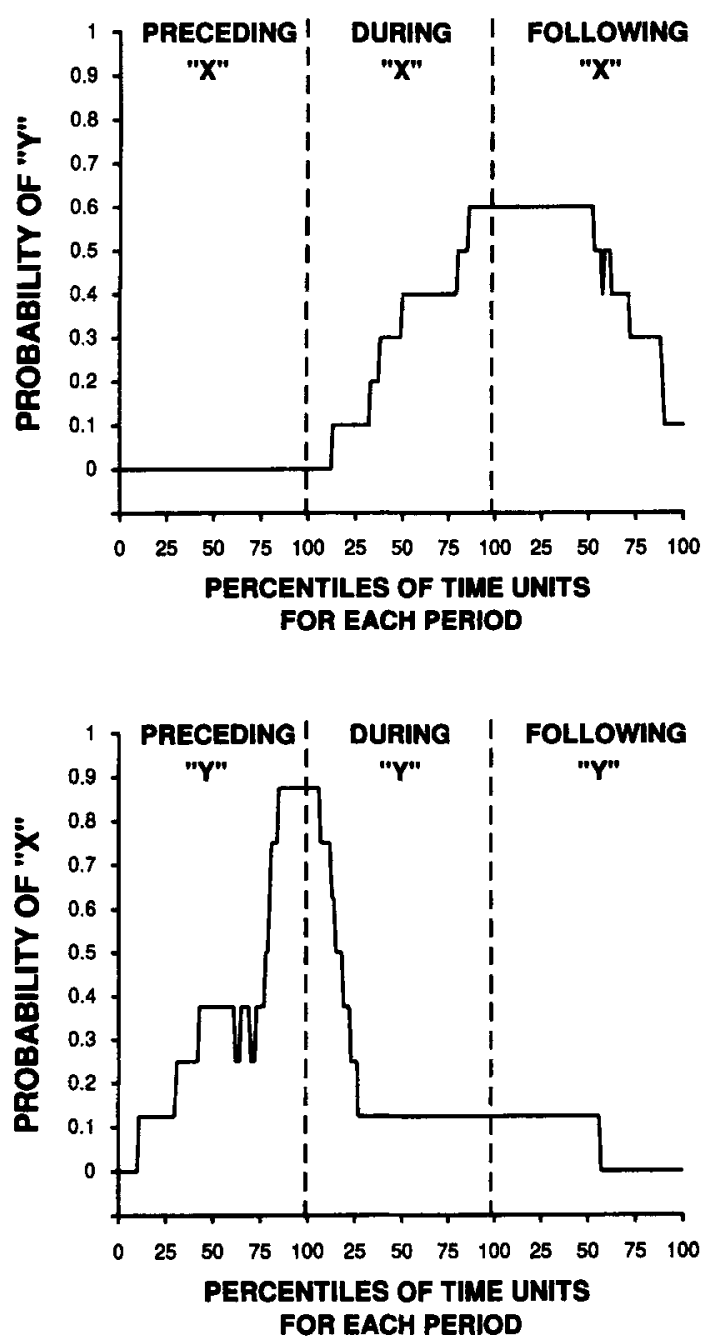

Figure 3. Plot of the mean probability of $\mathrm{Y}$ at each percentile preceding, during, and following $X$ (upper panel), and plot of the mean probability of $X$ at each percentile preceding, during, and following $Y$ (lower panel).
The profile in the upper panel of Figure 3 shows that the probability of $\mathrm{Y}$ remains at 0 preceding $\mathrm{X}$, increases from 0 to .6 during $X$, and decreases from .6 to about .1 following $X$. The profile in the lower panel of Figure 3 shows that the trend is almost the inverse of the upper panel in Figure 3 . Here, the probability of $X$ increases from 0 to about .9 preceding Y, decreases from about .9 to about .1 during $\mathrm{Y}$, and continues to decrease to 0 following $\mathrm{Y}$.

In order to quantify the profile in some way, a "profile score" for each period can be derived as follows. For each period of the profiles shown in Figure 3, the proportion of profile under the curve represents the probability of the target behavior occurring over that period. Similarly, the proportion of profile above the curve represents the probability of the target behavior not occurring over that period. For instance, for the preceding period of the profile in the upper panel of Figure 3, the proportion of profile under the "curve" is 0 , whereas the proportion of profile above the curve is 1.00 . Two cells of the familiar $2 \times$ 2 table for the period are now known (i.e., the probability of $Y$ preceding $X$ and the probability of not- $Y$ preceding $X$ ). To complete the table, the probability of $Y$ and not- $Y$ not preceding $X$ must be calculated. This is done by coding for the absence of the period preceding $\mathrm{X}$ and then conducting the normalized and pooled approach, nominating not-preceding- $X$ as the given behavior and $\mathrm{Y}$ as the target behavior. The proportion of profile under the curve over the during period of this profile thus represents the probability of Y not preceding X. Similarly, the proportion of profile above the curve over the during period represents the probability of not-Y not preceding $\mathrm{X}$. The table is now complete. A profile score for the period can now be calculated from the probability data in the $2 \times 2$ table, for instance, by use of the formula for Yule's $Q$ (see Bakeman \& Quera, 1995). Profile probability data and profile scores for each period of the profiles in Figure 3 are shown in Figure 4.

Interpreting the results in terms of the example behavior codes, mother vocalize appeared to be presented during child gaze and subsequently removed following child gaze. Conversely, child gaze appeared to be presented prior to mother vocalize and removed during and following mother vocalize (cf. conditional probability analysis above).

It should be stressed at this point, however, that the profile scores do not in any way determine the strength of a causal relationship. This is also true of Yule's $Q$ in the conditional probability analysis. It is quite possible, for instance, that $X$ and $Y$ in the example data are the results of another behavior, $Z$, with $Y$ simply having a larger latency time than $\mathrm{X}$. This possibility will be illustrated as follows.

Suppose the observer had recorded five more behaviors (e.g., Z, A, B, C, and D), in addition to X and Y. (The codes could represent additional behaviors of the child and/or its mother or even behaviors of additional participants.) The complete data file is shown at the top of the next page.

In order to determine whether $Z$ may be associated with $X$ and/or $Y$, behavior $Z$ could be nominated the given be- 


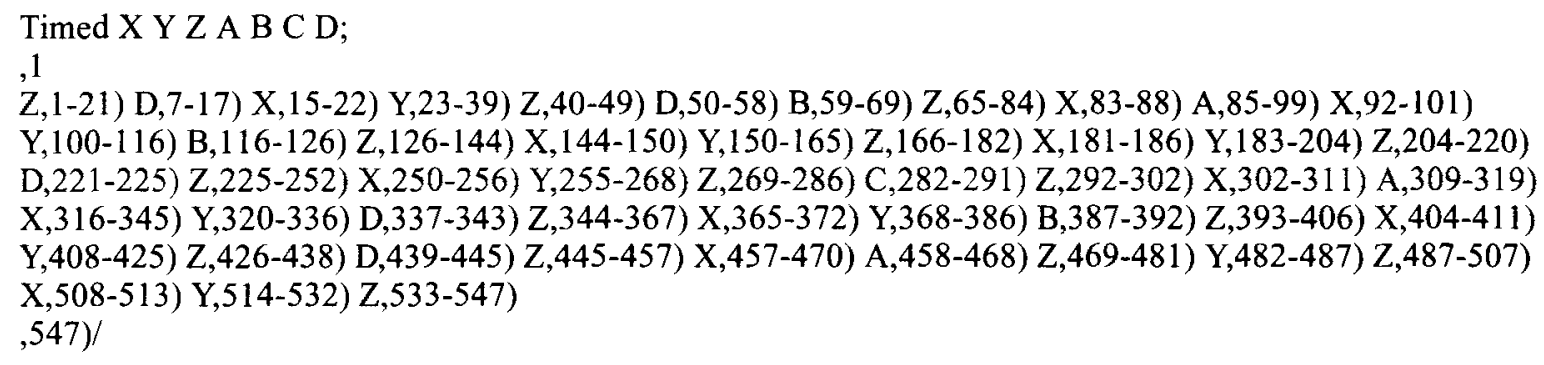

havior with behaviors $\mathrm{X}, \mathrm{Y}, \mathrm{A}, \mathrm{B}, \mathrm{C}$, and D nominated the target behaviors. The results of the analysis, using the normalized and pooled approach, are shown graphically in Figure 5.

The top left profile in Figure 5 shows that the probability of $X$ is low prior to $Z$, increases during $Z$, and is high immediately following $Z$, decreasing thereafter. This indicates an association between $Z$ and $X$ similar to the association between $X$ and $Y$ (cf. Figure 3, upper panel). This profile can be contrasted to the profile obtained between behavior $Z$ and behavior $Y$ (top right of Figure 5). Here, the probability of $Y$ decreases prior to $Z$, is at a low level during $Z$, and increases following $Z$. This indicates that $Y$ is most likely to occur at the midpoint between occurrences of $Z$. Profile scores for behaviors $X$ and $Y$ preceding, occurring during, and following $Z$ are shown in Table 1 , together with profile scores for behaviors A, B, $C$, and D preceding, occurring during, and following $Z$.

Although the analysis is still incomplete (we have not, for instance, examined the profile with $X$ as the given behavior and $\mathrm{Z}$ as the target behavior), the data serve to illustrate that the method is also useful in more complex situations. Indeed, the method has indicated that behavior $B$ may also be associated with $Z$ (see middle right profile of Figure 5, and Table 1). Of course, these results do not preclude the possibility that behaviors $X$ and $Y$ could be associated with yet another event that has not been monitored. The important point to note here, however, is that associations between behaviors can be graphically displayed and quantified in a meaningful way.

\section{Discussion}

We have devised a new method for conducting sequential analysis both in an attempt to overcome some of the difficulties associated with conducting a conditional probability approach to sequential analysis and to provide an accessible method of sequential analysis for clinicians. Essentially, by normalizing periods of time prior to, during, and following each occurrence of a nominated given behavior, the proportion of time units devoted to a target behavior can be estimated and then pooled across all occurrences of the given behavior. A summary diagram can then be constructed that represents the likelihood that the target behavior precedes, occurs concurrently
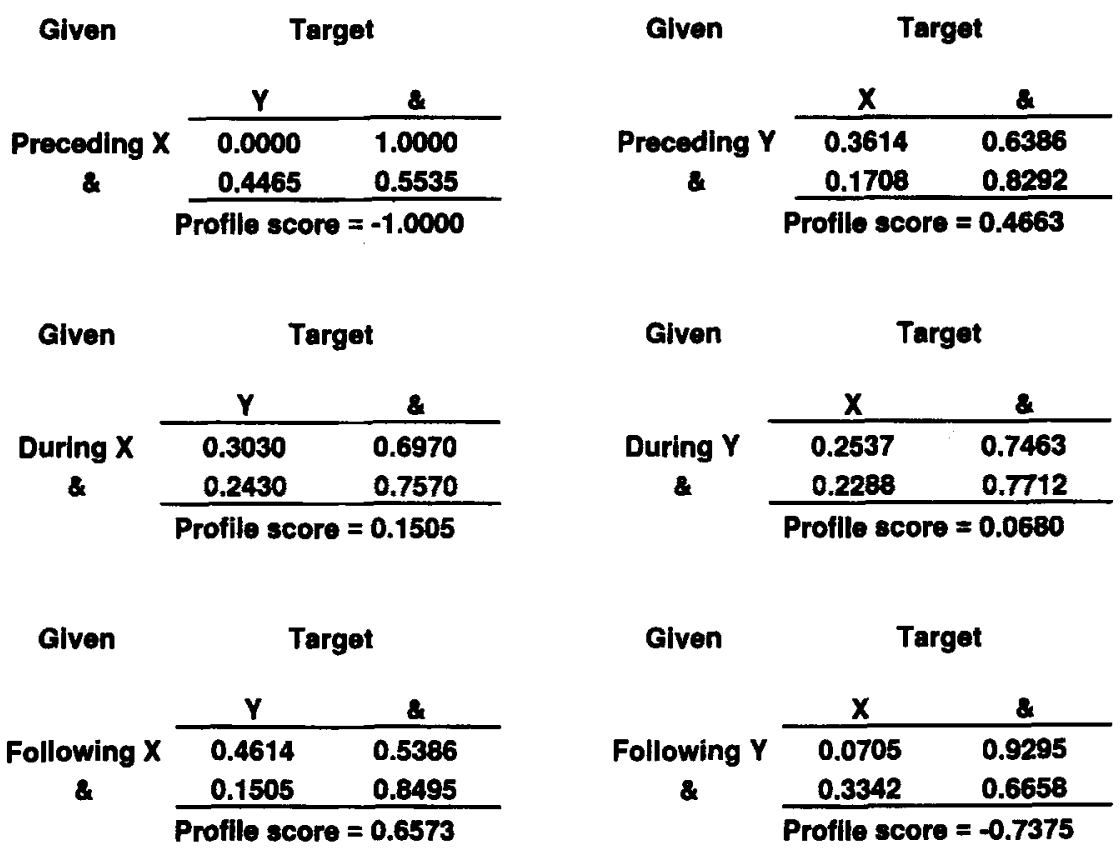

Figure 4. Profile data for each period of the profiles shown in Figure 3. Cells in the tables contain probability data over each period. Profile scores are shown below each table. 

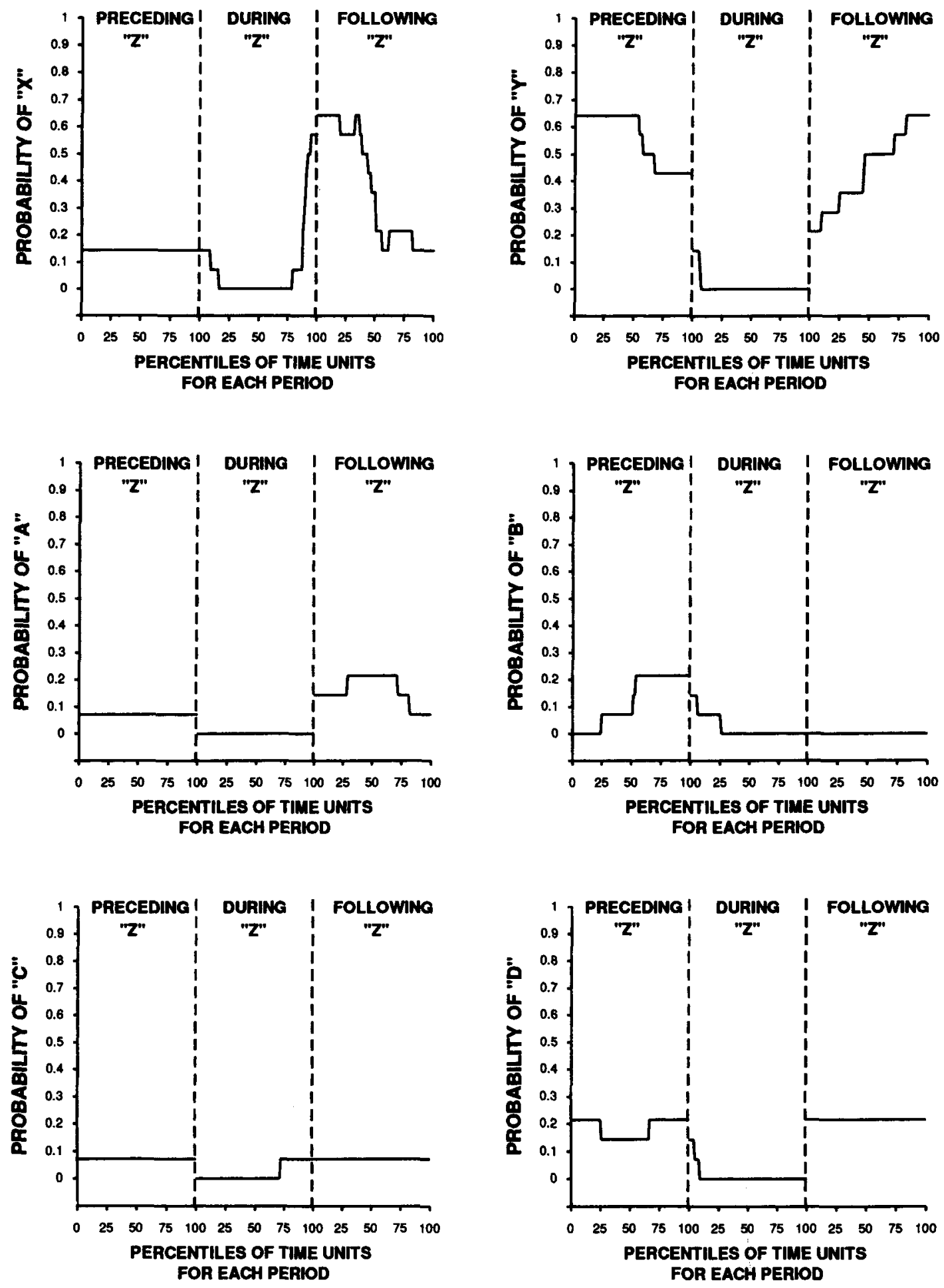

Figure 5. Plots of the mean probability of $X, Y, A, B, C$, and $D$ at each percentile preceding, during, and following $Z$.

with, and follows the given behavior. The normalized and pooled approach to sequential analysis therefore offers a relatively straightforward method of detecting and quantifying the temporal association between two behaviors.
We believe that the new method has a number of benefits for clinicians. First, the normalized and pooled method does not require the investigator to determine the duration of time windows, each of which may affect the 
Table 1

Profile Probability Data and Profile Scores

for Each Period of the Profiles Shown in Figure 5

\begin{tabular}{|c|c|c|c|c|c|c|}
\hline \multirow[b]{3}{*}{ Behavior Code } & \multicolumn{6}{|c|}{ Period } \\
\hline & \multicolumn{2}{|c|}{ Preceding $\mathrm{Z}$} & \multicolumn{2}{|c|}{ During Z } & \multicolumn{2}{|c|}{ Following $Z$} \\
\hline & Probability & Score & Probability & Score & Probability & Score \\
\hline $\mathrm{X}$ & .14 & -.24 & .09 & -.59 & .38 & .66 \\
\hline $\mathrm{Y}$ & .56 & .76 & .01 & -.97 & .45 & .50 \\
\hline A & .07 & -.02 & .00 & -1.00 & .16 & .80 \\
\hline B & .12 & .83 & .02 & -.53 & .00 & -1.00 \\
\hline $\mathrm{C}$ & .07 & .49 & .02 & -.60 & .07 & .49 \\
\hline $\mathrm{D}$ & .19 & .56 & .01 & -.93 & .21 & .73 \\
\hline
\end{tabular}

subsequent outcome and interpretation of the results. Second, because the method normalizes periods of time prior to, during, and following occurrences of behaviors, the problem of "overlapping," "truncated," and "null" windows is avoided and, hence, anomalies occurring in the analysis are also avoided (see Bakeman \& Quera, 1995). Although this problem can be corrected somewhat by splitting the time between occurrences of a given behavior when it is less than twice the desired window (see Appendix), this still requires careful manipulation of the data file. Third, the analysis summarizes the relationship between the two behaviors in one profile that, we believe, is intuitively appealing and easy to understand and interpret. Finally, elements of the profiles obtained from the method can be quantified to a profile score, which can then be subjected to further analyses.

This method of analysis also has a number of implications for studying social interactions. For instance, interpreting the results of the normalized and pooled approach in terms of the example behavior codes, it could be argued that the child's behavior served to occasion the behavior of its mother (see during and following periods of Figure 3, upper panel). Conversely, it could be argued that the mother's behavior served to remove the child's behavior (see during and following periods of Figure 3 , lower panel). If this interpretation is correct, it is likely that each participant in the interaction is influenced by the other's behavior (i.e., the child's behavior is influenced by the repeated presentation of the mother's behavior contingent on the child's behavior; similarly, the mother's behavior is influenced by the repeated removal of the child's behavior contingent on the mother's behavior). This relationship is indicative of the notions of "control and countercontrol" (Skinner, 1953) and "coercion" (Patterson, 1982; see also Oliver, 1995).

An additional benefit concerns the detection of intermittent responses to behaviors. Because the method described here determines the relative trend of one behavior occurring prior to, during, and following another behavior, sequences of behaviors that may occur reliably but also intermittently may also be detected. Thus, it is the profile that is produced from the data that may suggest a relationship between behaviors and not necessarily the significance of a conditional probability over its unconditional probability.
One disadvantage of the normalized and pooled method is that the approach appears to be applicable only to timed sequential data (TSD) (Bakeman \& Quera, 1995). Thus, data collected in other formats are not amenable to this method of analysis. Further refinement of the methodology described here, perhaps for use with other data formats, would therefore be warranted. Further problems with the method may also need to be investigated. For instance, the consequences of analyzing data files with small numbers of given behaviors is unclear. We believe that data files containing less than 10 occurrences of a given behavior would supply an insufficient number for analysis. (The example data contained small numbers of given behaviors for illustration purposes only.)

Furthermore, it is unclear whether longer time periods between successive occurrences of events are necessarily functionally equivalent to shorter ones, as the method implies (i.e., periods of 2 time units are treated as equivalent to periods of 100 time units). Indeed, the method also treats each "durational" time unit as functionally equivalent. This is also true of the conditional probability approach described here. It could be argued, for instance, that the onset of a behavior is more important than its duration. To investigate this possibility, the method could be adapted so that 2 time periods are defined rather than 3 (i.e., preceding, from the midpoint between the time unit following the offset of the $i$ th -1 occurrence and the time unit before the onset of the $i$ th occurrence, denoted $a_{1}$, to the time unit before the onset of the $i$ th occurrence, denoted $b_{1}$; and following, from the onset of the $i$ th occurrence, denoted $a_{2}$, to the midpoint between $a_{2}$ and the time unit before the onset of the $i$ th +1 occurrence, denoted $b_{2}$ ).

A final point concerns under what circumstances an investigator might choose to use the conditional probability approach or the normalized and pooled approach. Clearly, if an investigator wanted to determine whether or not a target behavior was more (or less) likely to occur within $x$ seconds of a given behavior, then the conditionally probability approach should be chosen. However, if an investigator wanted to determine whether or not a target behavior was either presented or removed relative to the occurrence of a given behavior, then the normalized and pooled approach should be chosen. Choosing the latter approach would also allow occurrences of 
a target behavior further removed in time from a given behavior to be included in the analysis.

In summary then, the methodology described here may provide a useful additional method with which to study social interactions. Indeed, the method may be particularly valuable to those without the statistical expertise or time to do a thorough conditional probability analysis. To this end, a computer program to conduct the normalized and pooled approach to sequential analysis is available from the authors free of charge. The program requires the user's observational data to be saved in TSD format. The user is simply required to specify the location of the data file(s) and to enter a given and a target behavior code. The program then outputs profile data and associated profile scores. The program runs on an IBM-compatible PC under Windows.

\section{REFERENCES}

Adamson, L. B., \& BaKeman, R. (1985). Affect and attention: Infants observed with mothers and peers. Child Development, 56, 582-593.

BAKEMAN, R. (1978). Untangling streams of behavior: Sequential analyses of observation data. In G. P. Sackett (Ed.), Observing behavior: Data collection and analysis methods (Vol. 2, pp. 63-78). Baltimore: University Park Press.

BAKEMAN, R., \& BRown, J. V. (1977). Behavioral dialogues: An approach to the assessment of mother-infant interaction. Child Development, 48, 195-203.

BaKeman, R., \& CASEY, R. L. (1995). Analyzing family interaction: Taking time into account. Journal of Family Psychology, 9, 131-143.

BAKEMAN, R., \& GoTTMAN, J. M. (1986). Observing interaction: An introduction to sequential analysis. Cambridge: Cambridge University Press.

BAKEMAN, R., \& QUERA, V. (1992). SDIS: A sequential data interchange standard. Behavior Research Methods, Instruments, \& Computers, 24, 554-559.

BaKeman, R., \& Quera, V. (1995). Analyzing interaction: Sequential analysis with SDIS \& GSEQ. Cambridge: Cambridge University Press.

BudEsCU, D. V. (1984). Tests of lagged dominance in sequential dyadic interaction. Psychological Bulletin, 96, 402-414.

DowdNey, L., MrazeK, D., Quinton, D., \& RutTer, M. (1984). Observation of parent-child interaction with two- to three-year-olds. Journal of Child Psychology \& Psychiatry, 25, 379-407.

Gardner, W., \& Griffin, W. A. (1989). Methods for the analysis of parallel streams of continuously recorded social behaviors. Psychological Bulletin, 105, 446-455.

GotTMAN, J. M. (1979). The consistency of nonverbal affect and affect reciprocity in marital interaction. Journal of Consulting \& Clinical Psychology, 48, 711-717.

GotTMan, I. M. (1981). Time-series analysis: A comprehensive introduction for social scientists. Cambridge: Cambridge University Press.

Gottman, J. M., Markman, H., \& Notarius, C. (1977). The topography of marital conflict: A sequential analysis of verbal and nonverbal behavior. Journal of Marriage \& the Family, 39, 461-477.

GotTman, J. M., \& RingLand, J. T. (1981). The analysis of dominance and bidirectionality in social development. Child Development, 52, 393-412.

GotTman, J. M., \& Roy, A. K. (1990). Sequential analysis: A guide for behavioral researchers. Cambridge: Cambridge University Press.

Hall, S. (1995). Review of the Observer 3.0. Journal of Child Psychology \& Psychiatry, 36, 1495-1498.

Hall, S., \& Oliver, C. (1992). Differential effects of severe selfinjurious behaviour on the behaviour of others. Behavioural Psychotherapy, 20, 355-365.

HigHLEN, P. S. (1986). Analysing patterns and sequence in counseling: Reactions of a counseling process researcher. Journal of Counseling Psychology, 33, 186-189.
Hill, C. E., Carter, J. A., \& O'Farrell, M. K. (1983). A case study of the process and outcome of time-limited counseling. Journal of Counseling Psychology, 30, 3-18.

Iacobucci, D., \& Wasserman, S. (1988). A general framework for the statistical analysis of sequential dyadic interaction data. Psychological Bulletin, 103, 379-390.

LERman, D. C., \& IWATA, B. A. (1993). Descriptive and experimental analyses of variables maintaining self-injurious behaviour. Journal of Applied Behavior Analysis, 26, 293-319.

LICHTENBerg, J. W., \& HeCK, E. J. (1986). Analysis of sequence and pattern in process research. Journal of Counseling Psychology, 33, 170-181.

MACE, F. C., \& LALLI, J. S. (1991). Linking descriptive and experimental analyses in the treatment of bizarre speech. Journal of Applied Behavior Analysis, 24, 553-562.

Moran, G., Dumas, J. E., \& Symons, D. K. (1992). Approaches to sequential analysis and the description of contingency in behavioral interaction. Behavioral Assessment, 14, 65-92.

Noldus, L. P. J. J. (1991). The Observer: A software system for collection and analysis of observational data. Behavior Research Methods, Instruments, \& Computers, 23, 415-429.

OLIVER, C. (1995). Annotation: Self-injurious behaviour in children with learning disabilities: Recent advances in assessment and intervention. Journal of Child Psychology \& Psychiatry, 36, 909-927.

Patterson, G. R. (1974). A basis for identifying stimuli which control behaviors in natural settings. Child Development, 45, 900-911.

PATTERSON, G. R. (1982). Coercive family process. Eugene, OR: Castalia.

Patterson, G. R., \& Forgatch, M. S. (1985). Therapist behavior as a determinant for client noncompliance: A paradox for the behavior modifier. Journal of Consulting \& Clinical Psychology, 53, 846-851.

REPP, A. C., \& FELCE, D. (1990). A microcomputer system used for evaluative and experimental behavioral research in mental handicap. Mental Handicap Research, 3, 21-32.

RepP, A. C., Harman, M. L., Felce, D., Van Acker, R. V., \& Karsh, K. G. (1989). Conducting behavioral assessments on computercollected data. Behavioral Assessment, 11, 249-268.

SACKETT, G. P. (1979). The lag sequential analysis of contingency and cyclicity in behavioral interaction research. In J. D. Osofsky (Ed.), Handbook of infant development (pp. 623-649). New York: Wiley.

SACKETT, G. P. (1987). Analysis of sequential social interaction data: Some issues, recent developments, and a causal inference model. In J. D. Osofsky (Ed.), Handbook of infant development (2nd ed., pp. 855-878). New York: Wiley.

Sackett, G. P., Holm, R., Crowley, C., \& Henkins, A. (1979). A FORTRAN program for lag sequential analysis of contingency and cyclicity in behavioral interaction data. Behavior Research Methods \& Instrumentation, 11, 366-378.

SkINNER, B. F. (1953). Science and human behavior. New York: The Free Press.

TAPP, J., WEHBY, J., \& ElLIS, D. (1995). A multiple option observation system for experimental studies. Behavior Research Methods, Instruments, \& Computers, 27, 25-31.

UNWIN, D. M., \& MARTIN, P. (1987), Recording behavior using a portable microcomputer. Behavior, 101, 87-100.

WiCKENS, T. D. (1993). Analysis of contingency tables with betweensubjects variability. Psychological Bulletin, 113, 191-204.

\section{NOTES}

1. These data and the data example presented later in the paper have been adapted from Repp et al., 1989, Behavioral Assessment, 11, p. 254. Copyright 1989 by Elsevier Science. Adapted by permission.

2 . We are grateful to Roger Bakeman for pointing this out.

3. A modified version of the method first appeared in Hall and Oliver (1992)

4. The first and last occurrences of $X$ are not included in the analysis because the number of time units from a previous occurrence of $X$ to the first occurrence of $X$ in the data and from last occurrence of $X$ in the data to a following occurrence of $\mathrm{X}$ are undetermined due to session boundaries.

5. We thank Roger Bakeman for his contribution in defining the time periods. 
APPENDIX

Modified Example Data and Commands Issued to Conduct the "Conditional Probability" Analysis Using GSEQ

Timed PREX X FOLX Y;

, 1

PREX,5-14) X, 15-22) FOLX,23-32) Y,23-39) PREX,73-82) X,83-88) FOLX,89-90) PREX,90-91) X,92-101)

Y,100-116) FOLX, 102-111) PREX,134-143) X,144-150) Y,150-165) FOLX,151-160) PREX,171-180)

$X, 181-186) \mathrm{Y}, 183-204)$ FOLX, 187-196) PREX,240-249) X,250-256) Y,255-268) FOLX,257-266)

PREX,292-301) X,302-311) FOLX,312-313) PREX,314-315) X,316-345) Y,320-336) FOLX,346-355)

PREX,355-364) X,365-372) Y,368-386) FOLX,373-382) PREX,394-403) X,404-411) Y,408-425)

FOLX,412-421) PREX,447-456) X,457-470) FOLX,471-480) Y,482-487) PREX,498-507) X,508-513)

FOLX,514-523) Y,514-532)

,547)/

File "xy";

Window PREY $=(\mathrm{Y}-10,(\mathrm{Y}-1$;

Window FOLY $=Y)+1, Y)+10$;

Stats CONP YULQ;

Target $Y$ \&;

Given PREX \&;

Target Y \&;

Given $X \&$;

Target Y \&;

Given FOLX \&;

Target X \&;

Given PREY \&;

Target X \&;

Given Y \&;

Target X \&;

Given FOLY \&;

End;

(Manuscript received January 30, 1996;

revision accepted for publication October 4,1996 .) 